\title{
Screening of quality markers of Jie-Geng decoction based on integration of multiple methods
}

\author{
Zhiyuan Fan ${ }^{1,2}$, Xue Han ${ }^{1,2}$, Lingling $X_{i a^{1,2}}$, Xiaohong $X u^{1,2}$, Jing $X_{i e}^{1,2}$, Quan \\ Zhang ${ }^{1,2}$, Qing Su ${ }^{1,2}$, Yanmei Sheng ${ }^{1,2}$, Xingliang $X_{i e^{1,2 *}}$ \\ ${ }^{1}$ School of Pharmacy, ${ }^{2}$ The Second Class Laboratory of Traditional Chinese Medicine Pharmaceutics, National Administration \\ of Traditional Chinese Medicine, Chengdu Medical College, Chengdu, China
}

*For correspondence: Email: 421733038@qq.com

\begin{abstract}
Purpose: To screen quality markers of Jie-Geng decoction (JGD) through multiple analytical methods and integration of network pharmacology and HPLC-ELSD fingerprint.

Methods: Network pharmacology was used to screen the potential bioactive components of JGD. Simultaneously, HPLC-ELSD fingerprint combined with multiple analytical methods was carried out for determination of the chemical compounds in JGD. Subsequently, quality markers for identification of quality variations in JGD were established through the integration of results from network pharmacology and fingerprinting, in combination with similarity analysis, hierarchical clustering analysis (HCA), and orthogonal partial least squares discrimination analysis (OPLS-DA).

Results: A total of 110 compounds responsible for the regulation of 36 target genes in airway inflammation and cough were identified through network pharmacology. Furthermore, 37 characteristic components were obtained through fingerprints. Similarity analysis revealed that the main bioactive compounds in the various batches of JGD were similar. Also, HCA and OPLS-DA analyses were performed to identify the potential quality markers. Glycyrrhizic acid, liquiritin, and platycodin D were selected as quality markers, based on effectiveness, measurability, and distinguishability. Furthermore, quality variations in JGD arose mostly from variations in origin of gancao.

Conclusion: The screened quality markers for JGD are useful in evaluation of factors that affect the quality and variation in JGD. The concept of integration of network pharmacology and fingerprint with multiple analytical methods might be a novel strategy for quality control of Traditional Chinese Medicine (TCM) formulations.
\end{abstract}

Keywords: Traditional Chinese Medicine, Quality marker, Jie-Geng decoction, Network pharmacology, Fingerprint

This is an Open Access article that uses a fund-ing model which does not charge readers or their institutions for access and distributed under the terms of the Creative Commons Attribution License (http://creativecommons.org/licenses/by/4.0) and the Budapest Open Access Initiative (http://www.budapestopenaccessinitiative.org/read), which permit unrestricted use, distribution, and reproduction in any medium, provided the original work is properly credited.

Tropical Journal of Pharmaceutical Research is indexed by Science Citation Index (SciSearch), Scopus, International Pharmaceutical Abstract, Chemical Abstracts, Embase, Index Copernicus, EBSCO, African Index Medicus, JournalSeek, Journal Citation Reports/Science Edition, Directory of Open Access Journals (DOAJ), African Journal Online, Bioline International, Open-J-Gate and Pharmacy Abstracts

\section{INTRODUCTION}

As is well known, Traditional Chinese Medicine (TCM) has a profound history of clinical application in the treatment and prevention of diseases. At present, decoction is still one of the major methods used in the clinical application of TCM formulations. Nevertheless, the quality of TCM formulation is unstable and susceptible to the influence of the medicinal materials, origin of 
TCM, and decoction process [1]. Therefore, stability has become a central issue in the quality control of TCM. Nowadays the quality control of TCM is usually carried out through a single content determination. However, the effective substances and mechanisms of action of many herbal prescriptions are still not very clear. Thus, it is difficult to objectively talk about the qualities of these drugs. Recently, quality marker (Qmarker), a new concept proposed by Changxiao Liu from the Chinese Academy of Engineering, was applied for quality and process control in TCM. Components that are present in TCM, as well as those generated during processing, are connected with the functional attributes of TCM. These components which have well-defined chemical structures, and can be qualitatively identified and quantitatively determined, are referred to as Q-markers [2]. In the determination of Q-markers, the first step involves the identification of the effective components of the herbs. Most TCM achieve therapeutic efficacy through multiple components and targets. Network pharmacology has gradually become an effectual research approach for determination of the complex biological relationships in TCM, including the interactions among herbs, compounds, targets, pathways, and diseases [3]. Jie-Geng decoction (JGD) is derived from TCM prescription consisting of $1: 2$ ratio of jiegeng (Platycodon grandiflorus (Jacq.) A. DC.) and gancao (Glycyrrhiza uralensis Fisch), and it has been widely used for the treatment of cough, asthma, pharyngodynia, and pulmonary abscess since the Eastern Han Dynasty in China [4]. Modern pharmacological research has shown that the main bioactive compounds in JGD are saponins and flavonoids which exert antiinflammatory and phlegm-removing effects $[5,6]$. Liu [7] reported that JGD produced protective effect against Staphylococcus aureus-induced acute lung injury (ALI). Furthermore, Tao [8] has reported that JGD protected mice against lipopolysaccharide-induced ALI through a mechanism involving anti-inflammatory effects of multiple targets of various compounds. The present study was aimed at screening Q-markers via the integration of network pharmacology and HPLC-ELSD fingerprint. Then, the Q-markers were used to assess variations in quality of JGD, as well as factors that influence the variations in JGD.

\section{EXPERIMENTAL}

\section{Reagents and materials}

Gancao and jiegeng were obtained from Sichuan Qianfang TCM Co. Ltd. Information on the origin of JGD samples are shown in Table S1. The reference standard of platycodin $\mathrm{D}$ was bought from the National Institute for the Control of Pharmaceutical and Biological Products. Liquiritin and glycyrrhizic acid ammonium salt were bought from Chengdu Mansite Biotechnology Co. Ltd. Liquiritin apioside, isoliquiritin apioside, neoisoliquritin, and liquiritigenin were obtained from Chongqing Gaoren Biotechnology Co. Ltd. The purity of each standard compounds was over $98.0 \%$. Acetonitrile (HPLC-grade) was obtained from Sigma-Aldrich Corporation, while water was purified using UPH-II-10T ultra-pure water production system. All other reagents were of analytical grade unless otherwise stated.

\section{Network pharmacology}

The compounds of gancao and jiegeng, and their molecular structures were obtained from the TCMSP database (http://tcmspw.com/tcmsp.php) and BATMAN-TCM database (http://bionet. ncpsb.org/batman-tcm/index.php/Home/Index /index). The potential bioactive compounds in JGD were selected via oral bioavailability (OB) and drug-likeness (DL) [9]. In this research, compounds with $\mathrm{OB} \geq 30 \%$ and $\mathrm{DL} \geq 0.18$ were screened as candidate bioactive constituents. In view of the fact that some compounds have welldefined pharmacological activities and higher contents, compounds with low OB or DL were also included. The targets of these compounds were collected from TCMSP, BATMAN-TCM, DrugBank (https://www.drugbank.ca/), TTD database (http://db.idrblab.net/ttd/), and CTD database (http://ctdbase.org/). Information on disease targets was obtained from GeneCards database (https://www.genecards.org/), the HPO database (https://hpo.jax.org/app/), and CTD database. The keywords/groups of words used to screen genes in these databases were "cough", "abnormal sputum" and "respiratory tract infection". Interactions among the common targets were analyzed using String (https://string$\mathrm{db}$. org/), and species were restricted to "homo sapiens" with a confidence score $>0.7$. Then protein-protein interaction (PPI) network was built using Cytoscape 3.5.1. "Network Analyzer" tools of Cytoscape were used to select the hub genes with values of "betweenness centrality", "closeness centrality" and "degree" greater than the median. Ultimately, a compound-target network was built using Cytoscape software.

\section{Sample preparation}

To prepare samples of JGD, $14 \mathrm{~g}$ jiegeng and 28 g gancao were soaked for $1 \mathrm{~h}$ in $600 \mathrm{~mL}$ of water, and boiled for $35 \mathrm{~min}$ in a casserole with lid until there was only $200 \mathrm{~mL}$ decoction left. 
After filtering through a 100-mesh screen cloth, the extract was concentrated to $40 \mathrm{~mL}$ under vacuum at $50{ }^{\circ} \mathrm{C}$, and thereafter converted to lyophilized powder in a freeze dryer at $-45^{\circ} \mathrm{C}$.

A mixed standard solution was obtained by dissolving each reference standard in methanol and diluting to desired concentrations. The concentrations of platycodin $\mathrm{D}$, liquiritin, glycyrrhizic acid ammonium salt, liquiritin apioside, isoliquiritin apioside, neoisoliquritin, and liquiritigenin in the mixed standard solution were $40,50,100,40,60,70$ and $55 \mu \mathrm{g} / \mathrm{mL}$, respectively. All solutions were filtered through a $0.45-\mu \mathrm{m}$ filter membrane prior to analysis.

To get the fingerprint solution, $2.0 \mathrm{~g} \mathrm{JGD}$ powder was extracted with $50 \mathrm{~mL}$ of $50 \%$ methanol using ultrasound (40 kHz and $250 \mathrm{~W}$ ) for $10 \mathrm{~min}$, and filtered. The filtrate was evaporated to dryness, and the residue was dissolved in $70 \%$ ethanol and left overnight in the fridge, followed by filtration and evaporation. The resultant residue was dissolved in $5 \mathrm{~mL}$ of $50 \%$ methanol and filtered through a $0.45-\mu \mathrm{m}$ membrane before fingerprint analysis.

\section{Solutions of Q-markers}

The contents of Q-markers were determined according to the method of Chinese Pharmacopoeia (2015 edition).

\section{Quantitative analysis using HPLC coupled with Ultraviolet detector (HPLC-UV)}

JGD powder $(0.1 \mathrm{~g})$ was diluted with $70 \%$ ethanol to $50 \mathrm{~mL}$, and the solution was subjected to ultrasonic extraction for about $10 \mathrm{~min}$.

\section{Quantitative analysis using HPLC coupled with evaporative light-scattering detector (HPLC-ELSD):}

JGD powder $(1.2 \mathrm{~g})$ was extracted with $50 \mathrm{~mL}$ of $50 \%$ methanol, and sonicated for $10 \mathrm{~min}$. The extract was then filtered and evaporated to dryness, and the residue was solubilized in 20 $\mathrm{mL}$ water, and extracted thrice with watersaturated n-butanol (20 mL each time). Next, 50 $\mathrm{mL}$ ammonia solution, and then $50 \mathrm{~mL}$ of $\mathrm{n}$ butanol saturated water were used to wash the n-butanol layers. The n-butanol layer was evaporated to dryness, and the residue was taken up in $5 \mathrm{~mL}$ methanol in a volumetric bottle.

\section{Fingerprint analysis using HPLC-ELSD}

Fingerprint analysis was performed on Dionex Utimate 3000 Series HPLC system (Thermo
Fisher, China) equipped with detector of ELSD 2000ES (Alltech, China). The chromatographic column was Phenomenex Luna C18 (2) (4.6 × $250 \mathrm{~mm}, 5 \mu \mathrm{m})$ at $30^{\circ} \mathrm{C}$. The mobile phase was composed of aqueous solution of $0.1 \%$ formic acid (eluent $\mathrm{A}$ ) and acetonitrile (eluent $\mathrm{B}$ ), and the gradient program used was $10-15 \%$ B ( $0-5$ $\min ), 15-19 \%$ B (5-25 min), 19-28 \% B (25-32 $\min ), 28-35 \%$ B (32-57 $\min ), 35-65 \%$ B (57-67 $\min ), 65-10 \%$ B (67-69 min), and 10\% B (69-75 $\min )$. The flow rate and the injection volume were $1.0 \mathrm{~mL} / \mathrm{min}$ and $10 \mu \mathrm{L}$, respectively. The parameters of ELSD 2000 ES were set with the model of impactor off, drift tube temperature of $108^{\circ} \mathrm{C}$ and $\mathrm{N}_{2}$ gas pressure of 13.8 psi.

\section{HPLC condition for quantitative analysis}

Quantitative analysis of liquiritin and glycyrrhizic acid was carried out with an Agilent 1260 Infinity (Agilent Technologies, Germany) equipped with variable wavelength detector. The separation was achieved on the same column used for fingerprint analysis. The mobile phase consisted of aqueous solution of $0.05 \%$ phosphoric acid (eluent A) and acetonitrile (eluent B). The gradient elution program was set as follows: 19 $\%$ B (0 - $8 \mathrm{~min}), 19-50 \%$ B (8 - $35 \mathrm{~min}), 50-$ $100 \%$ B (35 - $36 \mathrm{~min})$, and $100-19 \%$ B (36 - 40 min). The flow rate, column temperature, wavelength of ultraviolet spectra, and injection volume were $1.0 \mathrm{~mL} / \mathrm{min}, 30^{\circ} \mathrm{C}, 237 \mathrm{~nm}$, and 10 $\mu \mathrm{L}$, respectively.

Moreover, a quantitative analysis of platycodin D was conducted via the same instrument and column used for fingerprint analysis, using a mobile phase of water-acetonitrile (75: 25, v: v), column temperature of $30^{\circ} \mathrm{C}$, flow rate of $1.0 \mathrm{~mL}$ /min, and injection volume of $10 \mu \mathrm{L}$.

\section{Statistical analysis}

Similarity analysis was carried out using the Similarity Evaluation for Chromatographic Fingerprint of TCM (Version 2004A, China). This was used to calculate the correlation coefficients of similarity among 15 batches JGD and simulated fingerprint generated with similarity software based on common peaks from the 15 batches JGD.

Hierarchical clustering analysis (HCA) was used for classifying samples into clusters. Orthogonal partial least squares discrimination analysis (OPLS-DA) was used to predict the grouping of samples and maximize differences between classes. Moreover, HCA and OPLS-DA were done using SIMCA 14.1 software (Umetrics $A B$, Sweden). The common peak areas were used as 
input data. The Ward method was applied for $\mathrm{HCA}$. Data on Q-markers were analyzed using one-way analysis of variance (ANOVA) and the Tukey test or Fisher LSD test via OriginPro 2019 (version 9.6.5.169; OriginLab) software. Values of $p<0.05$ were considered indicative of statistically significant differences.

\section{RESULTS}

\section{Network pharmacology}

A total of 177 compounds in JGD were retrieved, comprising 158 compounds in gancao, and 19 compounds in jiegeng. These compounds had a total of 604 targets, while 751 targets of diseases (cough, abnormal sputum, and respiratory tract infections) were obtained from GeneCards, HPO, and CTD database after removing duplicates. A total of 110 common targets were shared between JGD and disease (Figure 1 a). A PPI network was constructed to elucidate detailed relationship among these communal targets (Figure $1 \mathrm{~b}$ ). A total of 39 hub genes (Figure $1 \mathrm{c}$ ) screened using topological metrics of "Betweenness centrality", "Closeness centrality" and "Degree", with their associated 111 candidate compounds, were obtained for further investigation.

A Compound-Target network was built to exhibit the relationship between the candidate compounds of JGD and hub genes (Figure $1 \mathrm{~d}$ ). This network comprised 148 nodes (2 herbs, 110 candidate compounds, and 36 targets) and 442 edges. Information on candidate compounds and targets are shown on Table S2. One compound and 3 targets were excluded for having no interaction with the whole network. As shown in Figure $1 \mathrm{~d}$, several compounds were obtained from gancao, while only a few compounds were obtained from jiegeng.

A total of 8 compounds with high degree values may play a crucial role in the network. Five of these compounds were from gancao i.e., kaempferol, quercetin, glycyrrhizic acid, naringenin, and liquirtin, while 3 compounds were from jiegeng i.e., platycodin D, luteolin, and acacetin. Thus, the 8 compounds with very high degree values in the network were screened as potential Q-markers. The top 4 targets of degree were PTGS2, PPARG, MAPK14, and AKT1, suggesting that these targets may be the key targets in the network.

\section{Results of similarity analysis}

The chromatographic fingerprint of 15 batches of JGD is shown in Figure 2 b. Peaks with acceptable resolution and reasonable heights were designated as common peaks. A total of 37 common peaks were labeled and a reference chromatogram was generated. Seven common peaks (Figure 2 a) were identified by matching their retention time (RT) values with those of reference compounds. Peaks $7,8,11,13,15$, 17 , and 30 were liquiritin apioside, liquiritin, isoliquiritin apioside, neoisoliquritin, liquiritigenin, platycodin D, and glycyrrhizic acid, respectively. The results from evaluation of similarity among 15 batches of JGD are shown in Table 1 and Table 2. The similarity values between the reference spectrum and 15 batches of JGD were all greater than 0.900 . Thus, there was similarity among the main chemical constituents of different batches of JGD.

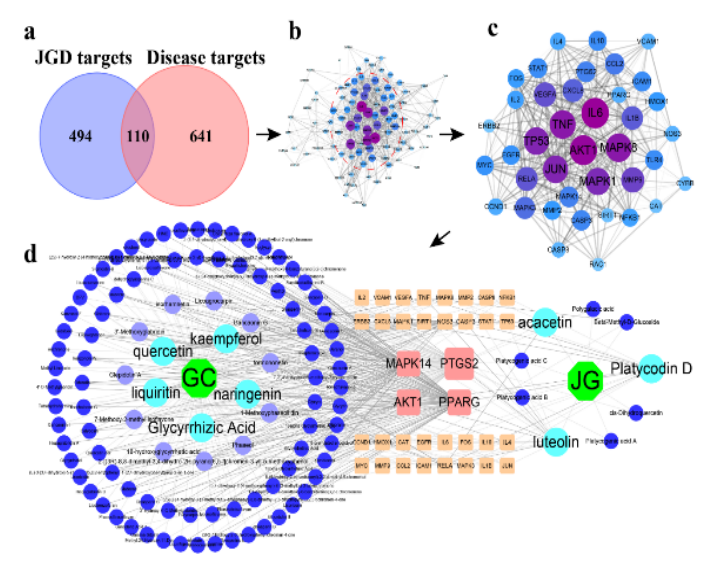

Figure 1: Network pharmacology. (a) Venn diagram of shared targets for JGD and disease. (b) PPI network of common targets. (c) PPI network of hub targets. The degree is represented with size and color: nodes with higher degree values are larger in size and purple in color. (d) Compound-Target network. The octagon, ellipse, and round rectangle represent herbs, compounds, and target genes, respectively. The degree is represented with size and color

\section{Results of HCA and OPLS-DA analyses}

The impact of different origins on JGD based on the areas of 37 common chromatographic peaks was determined using HCA. As shown in Figure $3 \mathrm{a}$, the samples (S1-S15) fell into three clusters: cluster I, cluster II, and cluster III. Samples from S12 and S13 formed cluster I; samples from S9, $\mathrm{S} 2$, and $\mathrm{S} 10$ formed cluster II, while the other samples formed cluster III. The result was consistent well with the fingerprint chromatograms, indicating a huge discrepancy among fingerprints of S12, S13, and the others, as marked with a red circle in Figure $2 \mathrm{~b}$.

In the OPLS-DA model, cross-validated predictive capability (Q2), variance (R2Y), and variance $(\mathrm{R} 2 \mathrm{X})$ of the model were $0.744,0.916$, 
and 0.876 , respectively. These three parameters were greater than 0.5 , implying that the model was suitable, and had good predictivity. The score plot of the OPLS-DA model is shown in Figure 3b. Chemical variables with variable importance in projection (VIP) value greater than 1 are considered to be important variables in classification [10]. In this study, there were 20 compounds with VIP values greater than 1 in Figure 3c. These comprised 3 known compounds (glycyrrhizic acid, liquiritin apioside, and isoliquiritin apioside) and 17 unknown compounds (peaks 26, 6, 32, 5, 37, 4, 33, 36, 31, $34,22,16,19,23,18,3$, and 12). These compounds with VIP scores greater than 1 were potential markers for quality control of JGD.

\section{Selected Q-markers}

Based on the results of network pharmacology, 8 compounds were screened out as key compounds capable of exerting pharmacological effects. One of these compounds, glycyrrhizic acid performed excellently in the HPLC fingerprint with VIP values greater than 1. Glycyrrhizic acid is not only an important component used for distinguishing different qualities of JGD, it also has anti-inflammatory properties. Therefore, it may serve as a Qmarker for JGD.

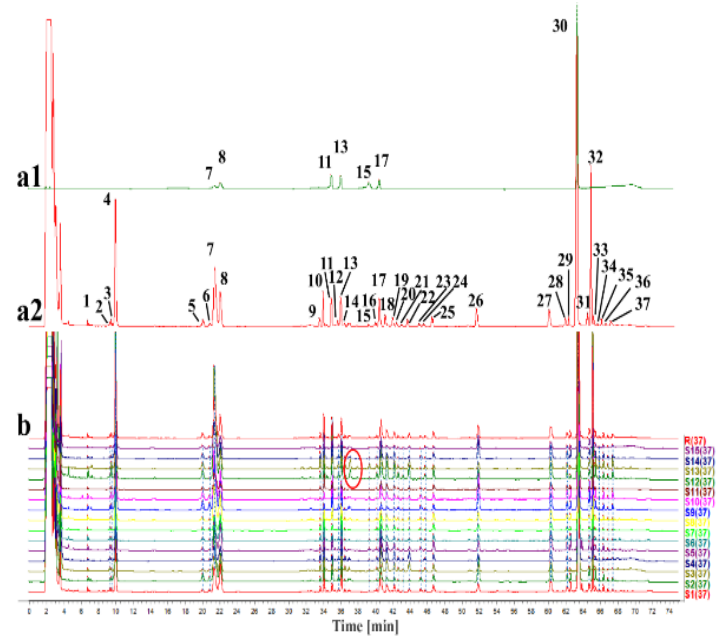

Figure 2: Chromatographic fingerprint of JGD. (a-1) Chromatogram of standard mixtures; $(\mathbf{a}-2)$ reference spectrum. In the chromatogram, peaks $7,8,11,13,15$, 17 , and 30 were liquiritin apioside, liquiritin, isoliquiritin apioside, neoisoliquritin, liquiritigenin, platycodin $\mathrm{D}$, and glycyrrhizic acid, respectively. (b) HPLC-ELSD chromatograms of 15 batches of JGD

However, considering the complexity of herbal formulas, a single Q-marker may not be enough. Taking into consideration the 7 known compounds from different batches of JGD in
HPLC fingerprints, and 8 key compounds obtained via network pharmacology, 3 compounds (glycyrrhizic acid, platycodin D, and liquirtin) were screened. It has been reported that platycodin D from jiegeng was exerted a variety of therapeutic effects, including antiinflammatory, apoptosis-inducing, airway mucin release-enhancing, and anti-cancer properties [11]. Studies have also shown that glycyrrhizic acid and liquirtin in gancao inhibited inflammatory response, decreased damage from oxidative stress, modulated apoptosis, and exerted antitumor effects [12]. Overall, glycyrrhizic acid, liquiritin, and platycodin D were chosen as Qmarkers for JGD, considering their effectiveness, measurability, and distinguishability.

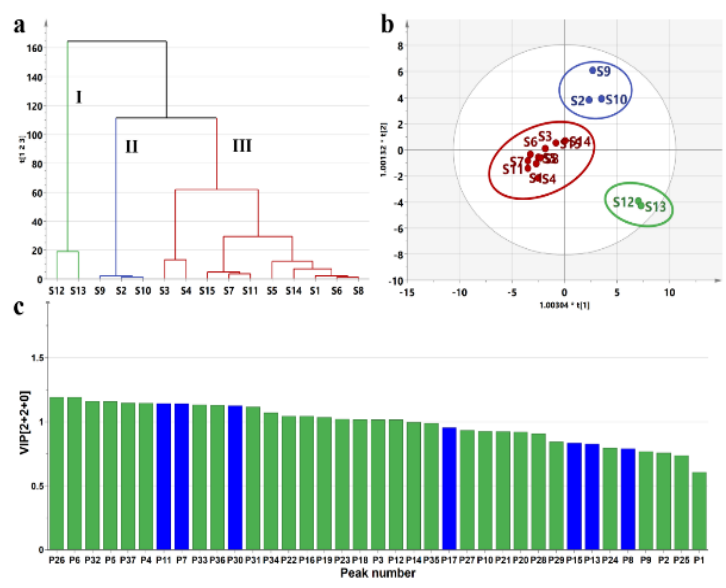

Figure 3: (a) HCA dendrogram. (b) OPLS-DA score plot. (c) VIP plot (the fingerprints of 7 reference compounds are colored blue)

\section{Quality variation in JGD}

Quality variations in TCM formula are of interest in the field of standardization. In this study, the contents of Q-markers in herbs and JGD were determined so as to ascertain quality variations in JGD. As shown in Table 1 and Table 2, the relative standard deviation (RSD) of transfers from herbs to JGD for liquiritin, glycyrrhizic acid, and platycodin D were $14.75,18.42$, and 15.28 $\%$, respectively. This demonstrates that the transfers of the three quality markers were stable during the process of preparation of 15 batches of jiegeng, and the differences between batches were small. In this study, the risk of quality variation in JGD due to the preparation process was relatively low, indicating that the process used in the preparation of JGD was stable and reliable. However, the RSD values for liquiritin, glycyrrhizic acid in gancao (52.08 and 24.46), and JGD (64.90 and 33.80) were much higher than those of platycodin D in jiegeng (19.98) and JGD (16.85), implying that the quality variation 
Table 1: Information on similarity values and the contents of Q-markers (S1 S8)

\begin{tabular}{|c|c|c|c|c|c|c|c|c|c|}
\hline \multicolumn{2}{|c|}{ SN } & S1 & S2 & S3 & S4 & S5 & S6 & S7 & S8 \\
\hline \multicolumn{2}{|c|}{ Similarity value } & 0.983 & 0.975 & 0.957 & 0.957 & 0.994 & 0.977 & 0.956 & 0.956 \\
\hline \multirow{3}{*}{$\begin{array}{l}\text { Liquiritin } \\
\text { (mg/g) }\end{array}$} & Gancao & 6.86 & 7.57 & 6.96 & 18.05 & 5.04 & 7.15 & 4.65 & 9.87 \\
\hline & JGD & 1.63 & 1.9 & 1.67 & 5.48 & 1.3 & 1.83 & 0.95 & 1.77 \\
\hline & Transfer (\%) & 23.74 & 25.06 & 23.99 & 30.35 & 25.71 & 25.68 & 20.39 & 17.89 \\
\hline \multirow{3}{*}{$\begin{array}{l}\text { Glycyrrhizic acid } \\
\text { (mg/g) }\end{array}$} & Gancao & 26 & 50.98 & 27.61 & 36.66 & 23.23 & 26.02 & 23.91 & 43.15 \\
\hline & JGD & 4.84 & 10.34 & 4.51 & 8.42 & 4.34 & 4.79 & 3.24 & 5.95 \\
\hline & Transfer (\%) & 18.6 & 20.28 & 16.34 & 22.96 & 18.7 & 18.39 & 13.56 & 13.79 \\
\hline \multirow{3}{*}{$\begin{array}{l}\text { Platycodin D } \\
(\mathrm{mg} / \mathrm{g})\end{array}$} & Jiegeng & 1.26 & 1.15 & 1.68 & 1.47 & 1.57 & 1.06 & 1.13 & 1.17 \\
\hline & JGD & 0.69 & 0.6 & 0.79 & 0.71 & 0.62 & 0.55 & 0.57 & 0.6 \\
\hline & Transfer (\%) & 54.67 & 52.07 & 46.92 & 47.96 & 39.76 & 51.38 & 50.83 & 50.85 \\
\hline
\end{tabular}

Table 2: Information on similarity values and the contents of Q-markers (S9 S15)

\begin{tabular}{|c|c|c|c|c|c|c|c|c|c|c|}
\hline \multicolumn{2}{|c|}{ SN } & S9 & $\mathrm{S10}$ & $\mathrm{S} 11$ & $\mathrm{~S} 12$ & S13 & S14 & $\mathrm{S15}$ & Mean & RSD (\%) \\
\hline \multicolumn{2}{|c|}{ Similarity value } & 0.963 & 0.96 & 0.94 & 0.942 & 0.951 & 0.993 & 0.991 & - & - \\
\hline & Gancao & 8.08 & 5.53 & 5.33 & 5.32 & 4.55 & 4.65 & 16.6 & 7.75 & 52.08 \\
\hline \multirow{2}{*}{$\begin{array}{l}\text { Liquiritin } \\
(\mathrm{mg} / \mathrm{g})\end{array}$} & JGD & 2.1 & 1.55 & 0.96 & 1.16 & 1.29 & 1.23 & 4.72 & 1.97 & 64.9 \\
\hline & $\begin{array}{l}\text { Transfer } \\
(\%)\end{array}$ & 26.04 & 28.06 & 17.97 & 21.76 & 28.42 & 26.56 & 28.43 & 24.67 & 14.75 \\
\hline \multirow{3}{*}{$\begin{array}{l}\text { Glycyrrhizic acid } \\
\text { (mg/g) }\end{array}$} & Gancao & 48.52 & 37.74 & 36.19 & 41.53 & 43.37 & 41.31 & 31.1 & 35.82 & 24.46 \\
\hline & JGD & 10.15 & 9.24 & 5.57 & 7.14 & 10.76 & 7.91 & 7.41 & 6.97 & 33.8 \\
\hline & Transfer (\%) & 20.92 & 24.49 & 15.39 & 17.2 & 24.81 & 19.14 & 23.82 & 19.23 & 18.42 \\
\hline \multirow{3}{*}{$\begin{array}{l}\text { Platycodin D } \\
\text { (mg/g) }\end{array}$} & Jie & 1.1 & 1.57 & 1.94 & 0.98 & 1.27 & 1.14 & 1.54 & 1.34 & 19.98 \\
\hline & JG & 0.4 & 0.62 & 0.85 & 0.6 & 0.8 & 0.62 & 0.68 & 0.65 & 16.85 \\
\hline & Transfer (\%) & 35.91 & 39.27 & 43.64 & 61.29 & 62.93 & 54.54 & 43.73 & 49.05 & 15.28 \\
\hline
\end{tabular}

among 15 batches JGD may result from herbs, especially gancao.

To further identify the factors that affect quality variation in gancao, the origin of gancao was taken into consideration. As shown in Figure 4, the contents of liquiritin in gancao from Gansu was significantly higher than that in gancao from Inner Mongolia $(p<0.05)$ or Xinjiang $(p<0.01)$.

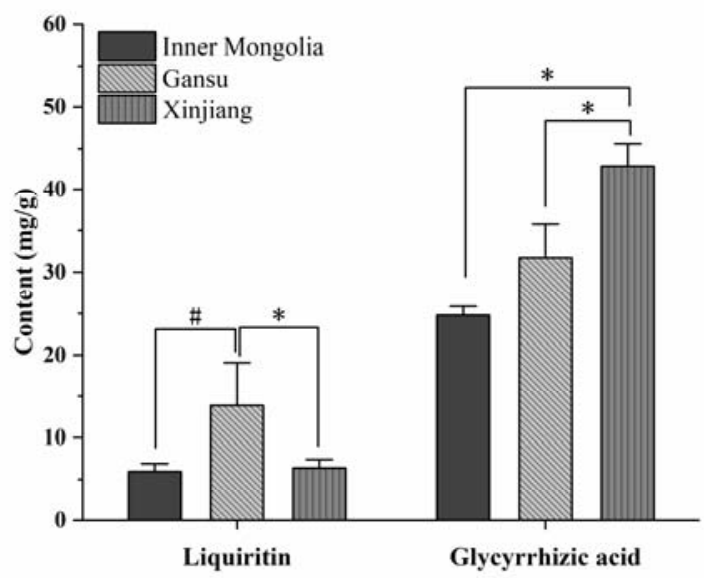

Figure 4: Contents of Q-markers in gancao from different origins. \# $p<0.05$, versus Gansu group; ${ }^{*} p<$ 0.01 , versus Xinjiang group

Moreover, the contents of glycyrrhizic acid from Gansu $(p<0.01)$ and Inner Mongolia $(p<0.01)$ were lower than that of gancao from Xinjiang, indicating that origin may be a significant factor that affects the quality variation in gancao. There were variations in the distributions of chemical constituents of gancao from different origins. Thus, the Q-markers can be used for quality evaluation of gancao by exploiting these discrepancies.

\section{DISCUSSION}

TCM has been in use for thousands of years in China, Japan, and Korea, due to their good clinical effects [13]. However, the unstable quality of traditional decoction and limited quality evaluation due to its complex ingredients hamper the curative effect of TCM. Therefore, the quality control of TCM is limited by its unstable quality and unclear mechanism of action. In the present study, the potential Q-markers associated with treatment of airway inflammation and cough were investigated via network pharmacology, and the Q-markers were defined using the results of chemometric analysis, to evaluate the quality variation in JGD.

In TCM classic formula Shang-han-Iun, JGD which is comprised of jiegeng and gancao, is widely used for treating lung diseases. In traditional use of TCM, jiegeng is the main herb that exerts curative effect, while gancao is a moderator that reduces the toxicity of JGD and improves its efficacy. Previous research has shown that gancao reduced inflammation when co-administered with jiegeng, and that 
glycyrrhizic acid from gancao also exerted antiinflammatory effects [8]. In this work, results from Compound-Target network revealed that most of the bioactive compounds came from gancao. Moreover, glycyrrhizic acid not only had higher degree values in the Compound-Target network, it also performed well, with larger VIP values in the OPLS-DA analysis. A total of 36 hub genes were obtained, and it was found that PTGS2, PPARG, MAPK14, and AKT1 which had high degree values in the Compound-Target network could be the key genes. Moreover, prostaglandin G/H synthase 2 (PTGS2) which had the highest degree value among the key genes, has been demonstrated to induce inflammation [14]. It is also the target of non-steroidal anti-inflammatory drugs (NSAIDs) [15]. This is consistent with the anti-inflammatory properties of JGD.

The Q-marker is a chemical substance that reflects the quality of Chinese herbal medicine, herbal portions, single prescription or formulas. It is widely used in the traceability system of quality process control. Zhao [16] screened Q-markers using the strategy of analyzing the transfer of compounds in shengmai injection, and applied the results for quality control. Moreover, Liao [17] applied chemical analysis and network pharmacology for screening Q-markers of Alisma orientale. In this study, network pharmacology and fingerprint coupled with multiple analytical methods were used to screen Q-markers which were applied for evaluation of quality variation in JGD. The results showed that there was little fluctuation in the transfer of Q-markers from herbs to JGD, while variation in herbs was much higher, notably in gancao. The contents of Qmarkers varied from region to region, suggesting that origin is a crucial factor that influences quality variation in related herbs. Therefore, to obtain a stable and consistent quality of JGD, the quality variation in medicinal materials, especially variation in quality of gancao due to source/origin, is a key risk point that needs to be paid special attention to and controlled.

\section{CONCLUSION}

Network pharmacology analysis of JGD has identified 110 compounds and 36 target genes related to airway inflammation and cough, suggesting that JGD contains multiple compounds with targets against the disease. Results from HPLC-ELSD fingerprint coupled with chemometric analysis of 15 batches of JGD demonstrated similarity among most of compounds from different batches. Results from evaluation of quality variations in herbs and JGD through Q-markers (glycyrrhizic acid, liquiritin, and platycodin D) screened using network pharmacology and chemometric analysis, revealed that variations in the quality of JGD were mainly due to origin/source of gancao.

\section{DECLARATIONS}

\section{Acknowledgement}

This work was supported by the Project of Sichuan Traditional Chinese Medicine Administration (no. 2018YY013) and the Fund Project of Sichuan Provincial Department of Education (no. 18ZB0160).

\section{Conflict of interest}

No conflict of interest is associated with this work.

\section{Contribution of authors}

We declare that this work was done by the authors named in this article and all liabilities pertaining to claims relating to the content of this article will be borne by the authors. The study was conceived and designed by Xingliang Xie, Yanmei Sheng, and Xue Han, and Xiaohong Xu. The experiment was done by Zhiyuan Fan, Lingling Xia, and Qing Su. The statistical analysis was carried out by Zhiyuan Fan, Jing Xie, and Quan Zhang. The manuscript was written by Zhiyuan Fan and revised by Xue Han and Xingliang Xie. All authors approved the final version

\section{Open Access}

This is an Open Access article that uses a funding model which does not charge readers or their institutions for access and distributed under the terms of the Creative Commons Attribution License (http://creativecommons.org/licenses/by/ 4.0) and the Budapest Open Access Initiative (http://www.budapestopenaccessinitiative.org/rea d), which permit unrestricted use, distribution, and reproduction in any medium, provided the original work is properly credited.

\section{REFERENCES}

1. Xie PS, Leung AY. Understanding the traditional aspect of Chinese medicine in order to achieve meaningful quality control of Chinese materia medica. J Chromatogr A. 2009; 1216(11): 1933-1940.

2. Liu C, Guo DA, Liu L. Quality transitivity and traceability system of herbal medicine products based on quality markers. Phytomedicine. 2018; 44: 247-257. 
3. Zhang Z, Yi P, Yang J, Huang J, Xu P, Hu M, Zhang C, Wang $B$, Peng $W$. Integrated network pharmacology analysis and serum metabolomics to reveal the cognitive improvement effect of Bushen Tiansui formula on Alzheimer's disease. J Ethnopharmacol. 2020; 249: 112371.

4. Shan JJ, Zou JS, XU JY, YU JH, Di LQ, Wang SC. Research advances of jiegeng tang. Chin J Exp Tradit Med Form. 2012; 18(19): 304-306.

5. Zhang $Q$, Ye M. Chemical analysis of the Chinese herbal medicine Gan-Cao (licorice). J Chromatogr A. 2009; 1216(11): 1954-1969.

6. Zhang $W$, Hou J, Yan X, Leng J, Li R, Zhang J, Xing J, Chen $C$, Wang $Z, L i$ W. Platycodon grandiflorum Saponins Ameliorate Cisplatin-Induced Acute Nephrotoxicity through the NF-kappaB-Mediated Inflammation and PI3K/Akt/Apoptosis Signaling Pathways. Nutrients. 2018; 10(9): 1328.

7. Liu Y, Hong Z, Qian J, Wang Y, Wang S. Protective effect of Jie-Geng-Tang against Staphylococcus aureus induced acute lung injury in mice and discovery of its effective constituents. J Ethnopharmacol. 2019; 243: 112076.

8. Tao J, Nie Y, Hou Y, Ma X, Ding G, Gao J, Jiang M, Bai G. Chemomics-Integrated Proteomics Analysis of JieGeng-Tang to Ameliorate Lipopolysaccharide-Induced Acute Lung Injury in Mice. Evid Based Complement Alternat Med. 2016; 2016: 7379146.

9. Seow V, Lim J, Cotterell AJ, Yau MK, Xu W, Lohman RJ, Kok WM, Stoermer MJ, Sweet MJ, Reid RC, et al. Receptor residence time trumps drug-likeness and oral bioavailability in determining efficacy of complement C5a antagonists. Sci Rep. 2016; 6(1): 24575.

10. Nguyen HT, Lee DK, Lee WJ, Lee G, Yoon SJ, Shin BK, Nguyen MD, Park JH, Lee J, Kwon SW. UPLCQTOFMS based metabolomics followed by stepwise partial least square-discriminant analysis (PLS-DA) explore the possible relation between the variations in secondary metabolites and the phylogenetic divergences of the genus Panax. J Chromatogr B Analyt Technol Biomed Life Sci. 2016; 1012-1013: 61-68.

11. Li W, Tian YH, Liu Y, Wang Z, Tang S, Zhang J, Wang YP. Platycodin $D$ exerts anti-tumor efficacy in $\mathrm{H} 22$ tumor-bearing mice via improving immune function and inducing apoptosis. J Toxicol Sci. 2016; 41(3): 417-428.

12. Yu JY, Ha JY, Kim KM, Jung YS, Jung JC, Oh S. AntiInflammatory activities of licorice extract and its active compounds, glycyrrhizic acid, liquiritin and liquiritigenin, in BV2 cells and mice liver. Molecules. 2015; 20(7): 13041-13054.

13. Cheung F. TCM: made in China. Nature. 2011; 480(7378): S82-S83.

14. Morham SG, Langenbach R, Loftin CD, Tiano HF, Vouloumanos N, Jennette JC, Mahler JF, Kluckman KD, Ledford A, Lee CA, et al. Prostaglandin synthase 2 gene disruption causes severe renal pathology in the mouse. Cell. 1995; 83(3): 473-482.

15. Harris RE, Beebe-Donk J, Doss H, Burr Doss D. Aspirin, ibuprofen, and other non-steroidal anti-inflammatory drugs in cancer prevention: a critical review of nonselective COX-2 blockade (review). Oncol Rep. 2005; 13(4): 559-583.

16. Zhao C, Liu H, Miao $P$, Wang $H$, Yu H, Wang C, Li Z. A Strategy for Selecting "Q-Markers" of Chinese Medical Preparation via Components Transfer Process Analysis with Application to the Quality Control of Shengmai Injection. Molecules. 2019; 24(9): 1811.

17. Liao M, Shang H, Li Y, Li T, Wang M, Zheng Y, Hou W, Liu C. An integrated approach to uncover quality marker underlying the effects of Alisma orientale on lipid metabolism, using chemical analysis and network pharmacology. Phytomedicine. 2018; 45: 93-104. 